\title{
A Cognitive Approach to Semantic Disorientation in Mandarin I - Theoretical Prerequisites and a Case Study*
}

\author{
Ting Yang \\ School of Foreign Languages, Chongqing College of Humanities, Science \& Technology, China
}

\begin{abstract}
Semantic disorientation refers to the phenomenon where sentence constituents with direct syntactic relations have no direct semantic linkage. The phenomenon is ubiquitous in Mandarin and related structures have been frequent research topics in the field of Chinese language study. However, there's no systemic description of their syntactic and semantic features, nor in-depth exploration of the linguistic and non-linguistic motivations. From the perspective of cognitive linguistics, the current study approaches this phenomenon with a usage-based and non-derivational language view. The phenomenon is defined and categorized on the cognitive and psychological basis and a descriptive and explanative frame-work is built for a more accurate and adequate account of the phenomenon. The syntactic and semantic features, as well as the linguistic and non-linguistic motivations of the disoriented verb-complement constructions are addressed as a case study.
\end{abstract}

Index Terms-semantic disorientation, cognitive linguistics, description \& explanation frame-work, verb-complement construction

\section{INTRODUCTION}

Generally speaking, syntactic forms have corresponding relationships with their semantic structures, and congruent ${ }^{1}$ pairs between forms and meaning are formed as a result. Therefore, the ultimate goal of language studies is to explore this form-meaning correspondence (Shao, 2007). However, syntactic forms and semantic structures may not correspond with each other in a completely neat way. For example,

(1) Xiaoming duguo le yige manchang de shujia.

Xiaoming pass-ASP ASP one-CL long LK summer holiday.

Xiaoming passed a long summer holiday.

(2) Xiaoming duguo le yige kuaile de shujia.

Xiaoming pass-ASP ASP one-CL happy LK summer holiday.

Xiaoming passed a happy summer holiday.

The syntactic patterns of (1) and (2) are exactly the same. But close examinations of their semantic structures show that they display different ways of form-meaning correspondence. Generally speaking, attributes modify their head words, just like "manchang" (long) in (1) does. The modifier provides temporal information for the summer holiday. However, the attribute "kuaile" (happy) in (2) does not modify the head word as the attribute in (1) does, even though they both have the same syntactic relation. Different from (1), "kuaile" (happy) and "shujia" (summer holiday) do not have direct semantic relation despite of their attribute-head syntactic formations. Instead, the attribute has close semantic linkage with the subject "Xiaoming". There seems to be a "mismatch" between the syntactic form and semantic structure in (2). Another similar example is as follows,

(3) Xiaoming kuaile de duguo le yige shujia.

Xiaoming happily LK pass-ASP ASP one-CL summer holiday.

Xiaoming happily passed a summer holiday.

Example (3) differs from (2) in that the adjective "kuaile" (happy) functions as an adverbial instead of being as an attribute. Still, the adverbial does not modify the verb "duguo" (pass) but has a modifying linkage with the subject "Xiaoming". The attempt to remove the modifier back into the syntactic slot that it seems to be belonged turns out to be a failure as the restored sentence can hardly be acceptable.

(4) kuaile de Xiaoming duguo le yige shujia.

Happy LK Xiaoming pass-ASP ASP one-CL summer holiday.

Happy Xiaoming passed a summer holiday.

\footnotetext{
* This paper is supported by the project "A Study of the Educational Reform of College English Major from the Perspective of Holistic Education" (19CRKXJJG11) by Chongqing College of Humanities, Science \& Technology. Abbreviations: ASP, aspect markers; CL, classifier; PRT, particle; LK, linker.

${ }^{1}$ The concept of congruence is borrowed from Systemic-functional Grammar.
} 
As is shown by (2) and (3), for the adjective "kuaile" (happy), no matter whether it functions as an attribute in (2) or as an adverbial in (3), its modifying relation with the subject "Xiaoming" never change. It seems that the attribute or the adverbial "belongs to" the subject and, because of certain reasons and syntactic managements, it is relocated. However, the restoration of the modifier back to its seemingly original place turns out to be a failure, as is shown by (4). The meaning changed dramatically from (2) and (3) as Xiaoming's happiness is irrelevant with his holiday, while in (2) and (3), Xiaoming's happiness is closely related with the summer holiday.

The "mismatch" as is shown in (2) and (3) caught linguists' attention and has been a frequent research topic since then. Scholars have proposed the Attribute View (Pan, 1981), Adverbial View (Zhu, 1982; Dai, 1982; Lu, 2001; Shao, 2002), Movement View (Lv, 1986) and Grammatical Metaphor View (Cong, 2013) for the syntactic and semantic gap as is shown by the sentences above. However, these views are easily falsified by examples like (4). The core belief of the views is that syntactic forms can be changed with their meanings being untouched. Therefore, all the views can be subsumed as a transformation view.

Structuralism holds that different morphological sequences represent different syntactic patterns, while cognitive linguistics believes that different sentences form different cognitive images in people's mind. Both of the linguistic approaches disagree with the transformation view.

The present study approaches this phenomenon from a psychological and cognitive perspective. The research shows that semantic disorientation is a pervasive linguistic phenomenon and the structures instantiating this phenomenon have been the research topic of various linguistic trends. The phenomenon will be defined and categorized on cognitive and psychological basis, and a descriptive and explanative frame-work will be built for a more accurate and adequate account of the phenomenon-related structures. The syntactic and semantic features, as well as their linguistic and non-linguistic motivations, of verb-complement constructions will be addressed as a case study.

\section{Definition AND CATEgoriZATION OF SEMANTIC Disorientation}

It is proposed that semantic disorientation refers to the non-congruence between syntactic forms and semantic structures (Zhang, 2005). The phenomenon is defined in the view of traditional linguistics, and it resides in the interface of syntax and semantics. Since cognitive linguistics holds that meaning is conceptualization and that syntactic forms correspond to conceptual structures, there is no "mismatch" or "non-congruence" between form and meaning. However, semantic disorientation indeed reflects a special linguistic phenomenon and has been the target of linguistic study for quite a long history. Therefore, the current paper tentatively defines semantic disorientation as a syntactic-semantic phenomenon where sentence constituents with direct syntactic relations have no direct semantic linkage. The definition follows the research tradition of the phenomenon and enables the current study to be a continuation and development of the previous studies in a consistent manner.

The definition limits the research area of the phenomenon within the interface of syntax and semantics, but it does not mean that it happens everywhere in the interface. Studies show that it most frequently occurs in the modifier-head and verb-complement constructions.

The definition of the phenomenon is based on cognition and psychology. Psychological researches show that when people are observing an object in the environment, they tend to single the object out as a perceptually prominent figure standing against the ground (i.e. the environment). Gestalt psychologists name it as the Figure-ground Theory and the introduction of the theory into linguistic researches produced profound effects. Two prominent features of the theory, figure-ground segregation and figure-ground reversal, are closely related with people's choice of linguistic structures and the subjective manipulation of syntactic sequences.

Figure and ground differ from each other when people are perceiving the world around them. The figure is singled out against the ground. It is coherent, structured, and has a form and shape. On the contrary, the ground is formless and unstructured. Visually, the figure stands out as an object against the background. Furthermore, the figure of perception might be changed on the condition that the candidate figure fulfills the Principles of Prägnanz, i.e., the principles of proximity, similarity, closure and continuation (Ungerer \& Schmid, 2006). Figure-ground segregation sheds light on the structures of language in that no matter on what syntactic level the units might be, some part may stand out as the figure, being more prominent than the others. Also, people may switch their emphasis based on communicative purposes and, most importantly, their ways of construal of the events or scenes in the world. It is important in the study of semantic disorientation in that the phenomenon is in fact a way of prominence switch and also reflects people's way of construal.

Figure-ground reversal is also the psychological foundation for semantic disorientation in that related structures are the linguistic manifestations in language. People may change their focuses of perception and, correspondingly, change the objects of prominence. Language structures follow the same principle of prominence. Semantically disoriented structures reflect people's change of profile in the construal of the events or scenes through syntactic managements.

It is true that semantic disorientation is pervasive in languages, but it does not mean that it exists in every aspect of language. As for Chinese, it is mainly about verb-complement constructions and modifier-head constructions. According to Bloomfield (1933), if the form-class of a constituent is the same with that of the structure, then the structure is an endocentric construction and that constituent is the head of the structure, otherwise, it is an exocentric construction. For verb-complement and modifier-head constructions, it is evidential that both of the constructions are endocentric because the verbs in the verb-complement constructions, and the nouns or verbs in the modifier-head 
constructions have the same form-classes with the whole constructions.

The function of the complements in the verb-complement constructions is to provide supplementary information for the verbs (Zhu, 1982; Ma \& Lu, 1997; Lu, 2016). They illustrate the location, time, extent and completement of the actions. For example,

(5) xiaoming chiwan le mifan.

Xiaoming eat-ASP ASP rice.

Xiaoming has finished the rice.

In (5), the complement "wan" indicates the completion of the action "chi" (eat). It semantically links with the verb. However, there are also cases where the complements do not link with the verbs directly but associate closely with other syntactic constituents.

(6) xiaoming dapo le huaping.

Xiaoming hit-broken ASP vase.

Xiaoming hit and broken the vase.

"dapo" (hit-broken) has been considered as a prototypical verb-result construction in Chinese and, thusly, been a repeated research topic. One of the most important points of the researches are the semantic orientation of the complement "po" as it directly links with the object of the sentence instead of the verb as the complement in (5) does. According to our definition, such kind of complements belongs to the semantic disorientation phenomenon.

The modifier-head constructions mainly consist of two types, attribute-noun constructions and adverbial-verb constructions. The constructions are considered as endocentric constructions as the modifiers in the constructions modify the heads, i.e. showing properties of the nominal heads or the way, extent, time, strength of the verbal heads. Usually, the modifiers directly link with the heads of the constructions, but there are cases where the modifiers are semantically linked elsewhere, which can be illustrated by example (2) and (3).

Semantic disorientation is not only pervasive in Chinese, it is also ubiquitous in other languages like English and Japanese. Jackendoff (1972), Keiichiro (1982), and Masayuki (1987) have noticed the semantic disorientation of the adverbials in the two languages. Although different languages differ in some aspects, the semantic disorientation could be categorized as follows,

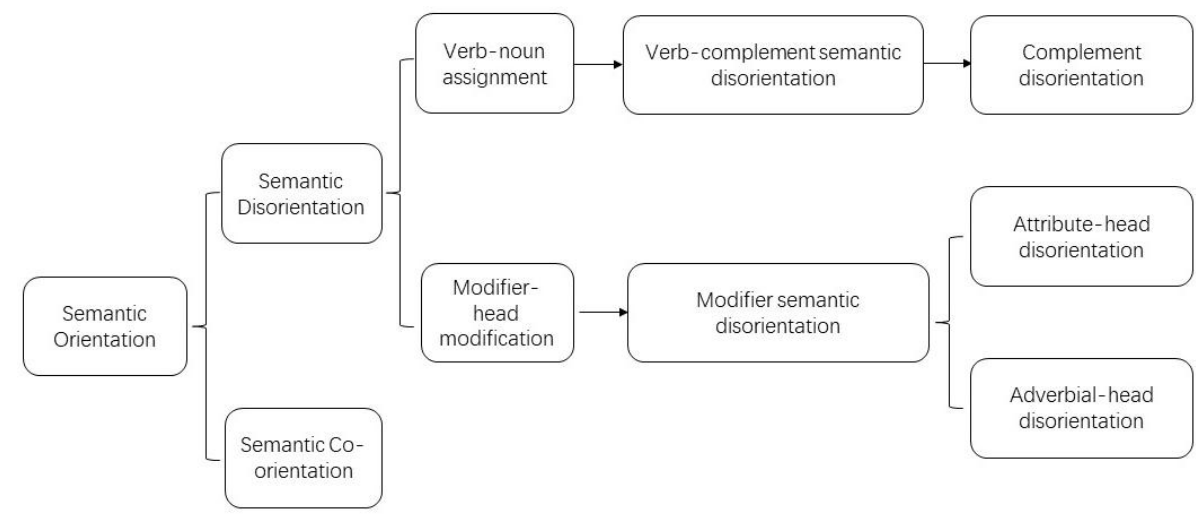

Figure I: Categorization of Semantic Disorientation in Mandarin

It can be easily told from Fig. I that there are two types of semantic disorientations, namely, verb-noun assignment and the modifier-head modification. The typical instantiation of the first type is the complement disorientation as illustrated by (5), while the prototypical modifier-head modifications are the attribute-head and the adverbial-head disorientation. It should be noted that the full picture of semantic orientation is far more complicated than what's shown by Fig. I. At least there are four types of semantic orientations as listed by Shen (2008), but only two of them are relevant to semantic disorientation. For convenience and clearance's sake, only the most relevant parts are listed here.

\section{The JustificATION AND COGNITIVE/PsychologiCAL BASIS OF THE CATEGORIZATION}

\section{A. Justification of the Semantic Disorientation Categorization}

The concept of semantic disorientation originates from the ingrained understanding of language being consisted of three levels, i.e. syntax, semantics and pragmatics. Through the long and slow evolution, languages developed a relatively stable syntactic-semantic correspondence. Different meanings are transferred by different syntactic forms and, vice versa, different syntactic configurations convey varying meanings. The phenomenon of semantic disorientation seems to be an exception in this regard since it demonstrates a kind of "mismatch" between form and meaning.

Usually, the complements and modifiers (including attributes and adverbials) provide supplementary information for the heads of the verb-complement and modifier-head constructions. However, as illustrated by (2) and (3), there are numerous cases where the complements and modifiers do not directly link with their syntactically adjacent elements. 
According to their syntactic roles and relation with the oriented parts, the current study classifies semantic orientation phenomenon into different categories as illustrated by Fig. I.

The categorization can be tested by word collocation requirements, sentence transformation and syntactic generalization.

The most straight-forward method is the judgement based on word collocation requirements, or simply, the language intuition by native speakers, no matter whether they are professionally trained for linguistic analysis or not.

(7) laowang xizizi de zha le pan huashengmi.

Laowang happy LK fried ASP CL peanuts.

Laowang happily fried a plate of peanuts.

(8) laowang cuicui de zha le pan huashengmi.

Laowang crisp LK fried ASP CL peanuts.

Laowang crisply fried a plate of peanuts.

People can easily tell that the adverbials in (7) and (8) do not semantically link with the verbs. Instead, the "xizizi" (happy) in (7) is semantically oriented to the subject, because the only human being, i.e. laowang, could have the feeling of happiness in the sentence. While the adverbial "cuicui" (crisp) in (8) can only link with the object "huashengmi" (peanuts). These judgements are simple, but deeply root in people's basic conceptualization of the selection and restriction of word collocations.

The second method of test comes from the transformation of the sentences. This method is based on the relevance of syntactic patterns (Lu, 2013). Different syntactic patterns may be internally linked despite of their sequential and formal differences.

(9) xizizi de laowang zha le pan huashengmi. $\rightarrow$ *laowang zha le pan xizizi de huashengmi.

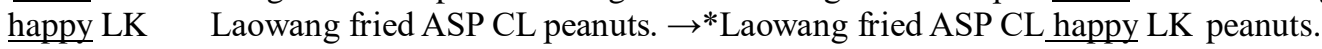

Happily Laowang fried a plate of peanuts. $\rightarrow$ *Laowang fried a plate of happy peanuts.

(10) laowang zha le pan cuicui de huashengmi. $\rightarrow{ }^{*}$ cuicui de laowang zha le pan huashengmi.

Laowang fried ASP CL crisp LK peanuts. $\rightarrow *$ crisp LK Laowang fried ASP CL peanuts.

Laowang fried a plate of Crispy peanuts. $\rightarrow$ * Crisply Laowang fried a plate of peanuts.

Syntactic elements cannot move freely in a sentence and it is clear that the sentences marked with "** in (9) and (10) could hardly be accepted because of their semantic weirdness. Also, there are other ways of syntactic transformation tests, like the $B a$ (把) sentences, Bei (被) sentences, verb-copying sentences, etc. The successful transformations from disoriented sentences are quite effective in the confirmation of the oriented targets of the complements or modifiers.

The third way of testing disorientation is from the perspective of sentence generalization. For transformation-generative linguists, the same surface structure may be generated from different deep structures. Therefore, sentence generalization can be employed as a way of disorientation test.

(11) na shou ge chang de Zhangsan leiliumanmian.

That CL song sing LK Zhangsan full of tears.

That song moved Zhangsan into tears. / Zhangsan sang that song and cried.

(11) is an ambiguous sentence because it can be understood as Zhangsan being the singer of the song or someone else being as the singer. The ambiguity can be analyzed as follows,
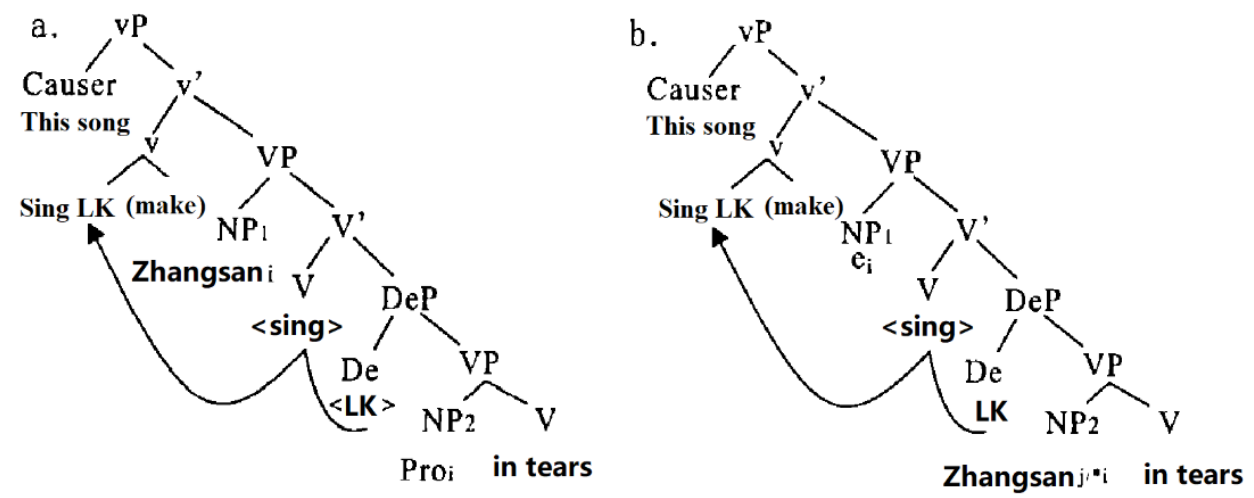

Figure II: Two Ways of Generalization

(Adapted from Jiang, 2006.)

From Fig. II, it is easy to tell the differences between the two ways of generalization and locate the root of the ambiguity. Comparatively speaking, this method is far more complicated than the previous two.

\section{B. The Cognitive and Psychological Basis of the Categorization}

Figure-ground Theory provides the psychological base for semantic disorientation. Gestalt psychologists find that when people are perceiving the world, they tend to single the things which catch their attention out against the 
environment. For example, when we are in a piano concert, our attention follows the sound of piano and, more often than not, neglects other musical instruments. Also, when we are watching high-jumping, our vision follows the track of the athlete and ignores the environment at the same time. People can also change their focus by switching their attention. These findings are illustrated by the vase/face illusion as follows.

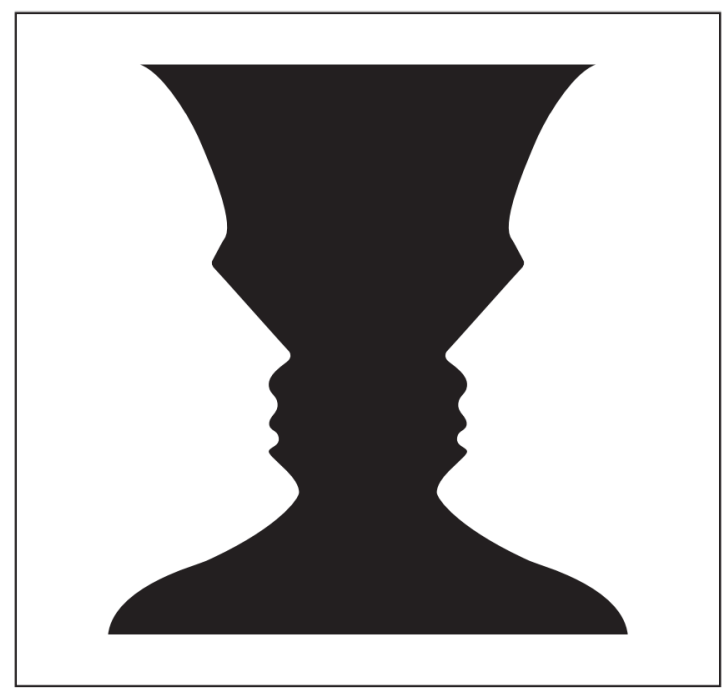

Figure III: The Face/vase Illusion

As is shown by Fig. III, if people focus on the black part, they will see a vase while if they switch their attention to the white part, they will notice the two faces. However, people cannot focus on the vase and faces at the same time. This psychological discovery is named as the figure-ground reversal. Its effects are also pervasive in language structures.

The current study categorizes semantic orientation into semantic disorientation and semantic co-orientation and points out that verb-complement, attribute-head and adverbial-head constructions are most likely to be semantically disorientated. The division of semantic disorientation and co-orientation is cognitively based.

As descriptive elements, complements provide supplementary information, e.g. time, speed, scope, extent, state, frequency, for the verbs (Zhu, 1982; Ma \& Lu, 1997). Therefore, it is an unmarked usage for action-related adjectives to be complements (Zhang, 2005, 2006; Shen, 2008). Similarly, as typical modifying elements in Chinese, attributes and adverbials are meant to provide descriptive information for their constructional heads.

The Endocentric Construction Theory proposed by Bloomfield (1933) holds that if the form-class of a certain constituent is the same with that of the construction, then the construction is endocentric and that constituent is the head of the construction. In actuality, the theory is founded on the Theory of Distributional Equivalence. Obviously, all of the three constructions are endocentric and, cognitively, the heads in the constructions are more prominent than other constituents as is shown by Fig. IV.

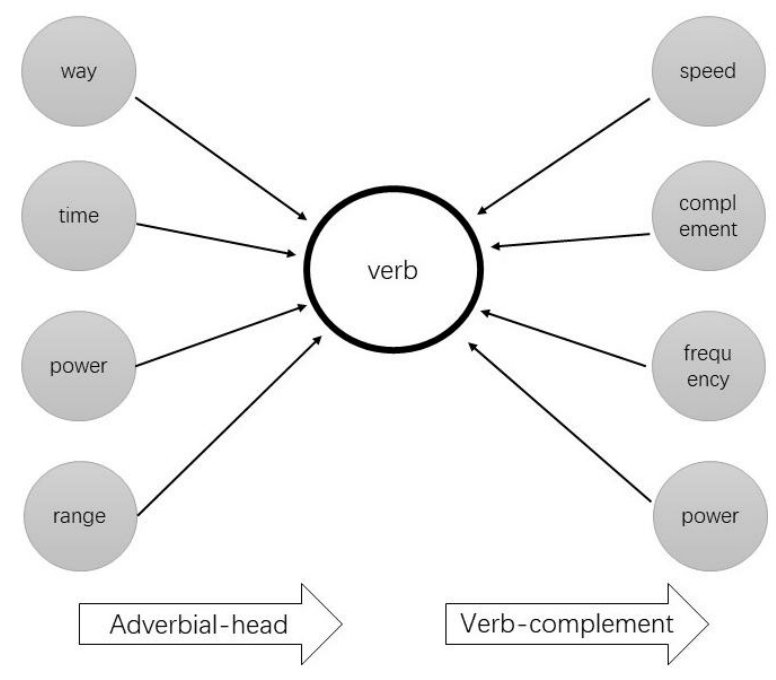

Figure IV: The Profile of Semantic Co-orientation Constructions

As is shown by Fig. IV, no matter whether they are adverbial-head or verb-complement configurations, the heads 
stand out as the figures (marked with bold-lined circle) of the semantic co-orientation constructions and the other elements act as the ground (marked in grey).

With the development of Chinese study and especially with the introduction of cognitive linguistics, the above-mentioned view is questioned by scholars like Li (1984), Gu (1992), Yuan (2001), Croft (2001) etc. With regard to the heads of verb-complement constructions, the scholars hold that, instead of the verbs, the complements are more likely to be the heads. Complement Head Theory and the Division of Syntactic-semantic Head Theory are proposed to explain why the complements bear higher information density. Croft (2001) and Shen (2004) also questioned the plausibility of the application of the Endocentric Theory to the confirmation of the heads in the verb-complement constructions.

The above-mentioned questions are in accordance with native speakers' language intuition and the core of the problem lies in the ubiquitous distriution of disoriented constructions. From the perspective of cognition and psychology, the essence of semantic disorientation is the reversal of the figure-ground relation in the constructions as is shown by Fig. V.



Figure V: The Reversal of Figure-ground Relation in Disoriented Constructions

As is shown by Fig. V, the original figure (i.e. the verb) becomes less prominent and acts as the ground in the relation (marked in grey), while the disoriented adverbials and complements function as the figures in the relations because of their higher information density and cognitive prominence. They are heavily loaded with information like speaker subjectivity, intention, evaluation, etc.

As for disoriented complements, they have their own argument structures, and dominate or share arguments with the verbs. Instead of providing supplementary information for the events being constructed by the verbs as the co-oriented complements do, disoriented complements construct resultative or evaluative events by themselves and, in terms of prominence, they are far more important than the causitive or conditional events constructed by the verbs. In Talmy's terms (2000), the disoriented complements build a framing event while the verbs only construct a co-event, whose main function is to provide environmental or supplemental information.

\section{CONSTRUCTING A DESCRIPTIVE AND EXPLANATIVE FRAME-WORK}

\section{A. The Basic Principles of Constructing the Frame}

The current study follows the research paradigm of cognitive linguistics and, at the same time, combines traditional semantic analysis with frame semantics, event structure theory, figure-ground theory, and construal theory. Based on the usage-based approach, and the surface-generalization and non-derivational view from construction grammar, it intends to construct a frame-work capable of accurate description and efficient explanation.

1. Usage-based Approach

Since the middle of last century, linguists have been concerned with the influence of language use on language structures. They attached great importance to real world utterances and their impacts on language evolution, grammaticalization, and children's language acquisition. These linguists include T. Givon, S. A. Thompson, P. Hopper, R. Langacker, L. Talmy, G. Lakoff, G. Fauconnier, J. Taylor, etc., and their research paradigms can be collectively referred to as a Use-based Model.

The usage-based approach or usage-based language view believes that language knowledge is built on the basis of human physiological conditions, cognitive mechanisms and the high frequency of linguistic events; language knowledge is the sum of linguistic structures, which are symbolic units of form and meaning with different levels of 
complexity and abstraction. The core of the claim is that language is a usage-based, complex, and dynamic cognitive adaptation system. There are five major tenets as listed below.

(1) The fundamental purpose of language is communication;

(2) Natural language is used in and affected by context, e.g., the relationship between the speaker and the listener influences the choice of words;

(3) Language is acquired, and there is no innate language acquisition mechanism;

(4) Meanings do not only come from morphological terms, but also from linguistic structures;

(5) No differentiation is made between syntax and morphology. Each syntactic structure has its unique meaning, and there is no conversional relationship between each other, e.g., passive sentences are not converted from active sentences.

The major difference between the usage-based language approach and previous research views or paradigms is the ultimate emphasis on natural utterance and its relation with grammatical structures.

2. Surface Generalization and Non-derivation View

Goldberg $(2006,2013)$ points out that surface generalization and non-derivation are the two important principles of Construction Grammar. Surface generalization emphasizes the importance of surface form and believes that each grammatical form has its unique function. The generalization of surface form is more extensive than generalizations derived from deduction or conversion. On the other hand, the non-derivation view negates all formal conversions and derivations, and holds that conceptual structures are directly projected to syntactic structures.

Surface generalization and non-derivation views are mainly used in the study of linguistic constructions, and are also considered as the basic views of cognitive linguistics at large. They function as the cognitive basis for semantic disorientation too. The current study holds that sentences stand as holistic structures with unique meanings and form different images in people's mind. The claims are incompatible with the transformation view proposed by other research schools. From the dynamics of cognitive construal, different linear sequences mean completely different conceptual structures and psychological access paths, which are the consequences of different ways of construal on the same events.

\section{B. A Framework for Semantic Disorientation Description and Explanation}

In line with the research paradigm of cognitive linguistics and based on the usage-based approach and surface generalization and non-derivational view, the article intends to build a descriptive and explanative framework for semantic disorientation phenomenon.

Linguistic description methods can be divided into three categories: Classified Description, Layered Description and Feature Description ( $\mathrm{Du}, 2020)$. These methods cannot be used freely since they have specific requirements on the research objects. The objects described by Classified Description must have collective features and high degrees of homogeneity within the group and sufficient discrepancy from adjacent categories. While the Layered Description method can only be applied to subjects whose collective inner structures are hierarchical. As for semantic disorientation phenomenon, all the three methods are applicable.

Scientific explanation mainly includes deductive explanation and model explanation (Du, 2020). Linguistic explanation is an explanative method based on both deductive explanation and model explanation. Linguistic explanation can be carried out from three dimensions, namely, historical, grammatical and functional. As to semantic disorientation, model explanation is mainly used in the exploration of its cognitive motivations.

Therefore, a description and explanation framework can be proposed as is shown by Figure VI.



Figure VI: The Descriptive and Explanative Framework of Semantic Disorientation 
As is shown by Fig. VI, the framework starts from the judgement of orientation types which are classified into two categories and three sub-classes. Then the syntactic and semantic features are examined successively. As for the syntactic features, the disorienting elements are surveyed from their morphological features, distribution, syntactic sequences and quantifiers etc. for contrastive purposes. Then, the semantic features of the disoriented constituents are examined. "Three dimensions" refer to the targets, the number of targets and the collocation with the targets. Furthermore, their semantic types, information structures and pragmatic effects are surveyed in this regard.

The accuracy of description and adequacy of explanation are the ultimate goals of linguistic study, with the former being the premise of the adequate explanation. Therefore, the motivations of the disoriented structures are explored from linguistic and non-linguistic aspects. The usage of the disoriented structures may be motivated meta-linguistically, pragmatically, socially, or cognitively, and it is also possible that different types of motivations may function together.

\section{Verb-COMPlement CONSTRUCTIONS In MANDARIN: A CASE Study}

\section{A. Syntactic and Semantic Descriptions of the Verb-complement Constructions}

Based on the descriptive and explanative frame-work as constructed in Part IV, verb-complement constructions are described and explained as a case study. In a verb-complement construction, if the semantic orientation target of the complement is the verb, then it is a semantic co-orientation construction, otherwise it is a disorientation construction. Co-orienting complements are investigated and it is found that they have unique syntactic and semantic features, e.g., having looser restrictions on the syllables and being more likely to be grammaticalized. For example,

(12) Disyllables: zou shangqu (walk-up), pao chulai (run-out), kan xiaqu (watch-downward)

Monosyllabic: pao diao (run-lose), tuchu (vomit-out), chiwan (eat-over)

In (12), both disyllabic and monosyllabic complements are used in the verb-complement constructions and provide information, like direction, completeness, etc. for the verbs. It is also found that the co-orienting complements are more likely to be grammaticalized than the disorienting ones. Despite of their different degrees of grammaticalization, they have higher possibilities of losing their concrete meaning and taking on abstract grammatical functions.

Let's turn to the syntactic and semantic features of the disorienting complements. Despite of the differences as listed previously, the information structures and argument structures of the disorienting complements are dramatically different from those of the co-orienting complements.

As for the information structure, disorienting complements do not provide any periphery information to the verbs. The complements denote a framing event while the verbs build a co-event. Therefore, two events are expressed through one disorienting verb-complement construction rather than only one event as in a co-orienting verb-complement construction. It can be safely concluded that disoriented verb-complement constructions have higher information density than co-oriented verb-complement constructions.

As for the argument structures of the verb-complement constructions, co-orienting complements are more likely to be grammaticalized and take the verbs as their semantic orienting targets. Co-orienting complements can hardly dominate any argument. Things are different with regard to the disorienting complements as they construct framing events and have their own or share arguments the verbs.

The survey of the argument structures (mainly the number of the arguments) of the disorienting complements shows that the number of arguments in the sentences is exactly the same with the valence of the verbs or adjectives that function as the complements. The findings also justify the value of the reductionist view of argument structure analysis in verb-complement constructions.

\section{B. Motivations of the Verb-complement Constructions}

The motivations of the language structures can be grouped into linguistic motivations and non-linguistic motivations as is shown by Fig. VI. Verb-complement constructions are motivated both linguistically and non-linguistically.

Linguistic motivations can be explored both diachronically and synchronically. Diachronically, Chinese language has gone through a process of disyllabicalization and monofocallization. In ancient Chinese, there can be two focuses in one sentence. For example,

(13) gong er po zhi.

attack LK break it (the city)

Attack and break the city.

(14) ji er sha zhi.

hit LK kill it (the people)

Hit and kill the people.

As is shown by (13) and (14), ancient Chinese sentences usually have two focuses. With the linguistic evolvement, the two verbs link together and some of them even become compound words. Still, the inner structure of the words can be told easily. Language evolvements are slow and it is estimated that the verb-complement constructions come into being about hundreds of years ago (c.f. Shi, 2003).

Non-linguistic motivations are more complicated. The most common non-linguistic motivations are social motivations (like politeness, tradition, etc.), psychological motivations, and cognitive motivations. Not every language structure is motivated by all the factors and the motivations do not function in the same way. The psychological and 
cognitive motivations of the constructions are analyzed as a case study.

Gestalt psychologists find the intriguing illusion of the face-vase picture as is shown by Fig. III and people's special way of perceiving the world. People may single one thing out from its environment, and focuses on it. The thing that is being singled out is named as the figure. It is structured, has closed line and stands against the background, which is usually unstructured, uniform and formless. Psychologists call this as the figure-ground segregation. Also, people may change their chosen figure, and focus on other things in the background on the condition that the new figure fulfills the Principles of Prägnanz. It means that the figure-ground relation is reversible.

The psychological findings motivate the emergence of disoriented verb-complement constructions. It is true that the verb-complement constructions are form-meaning pairs, still they are reductive in the reductionist view. From the co-oriented constructions to the disoriented constructions, people change the roles of the verbs and complements in the figure-ground configuration. In the co-oriented constructions, the verbs are the figures of the configuration while the complements provide supplementary information for the verbs. While in the disoriented constructions, the complements dominate arguments by themselves and, most importantly, they construct the framing events of the sentences while the verbs denote the causative or conditional co-event. Of course, result is far more important than the reasons and conditions. Therefore, the complements in the disoriented verb-complement constructions step out from the background and are being perceived as the figures.

\section{CONCLUSION}

The current study explores the theoretical prerequisites for the analysis of semantic disorientation phenomenon in Mandarin. It is an all-new attempt to take this phenomenon as a research subject despite of the fact that the relevant structures have been repeated research topics with quite a long history. It is demonstrated that the categorization of the disorienting structures is cognitively and psychologically based and there are syntactic as well as semantic methods to test the validity of the categorization. Based on the usage-based approach and with the surface generalization and non-reductionist view, a descriptive and explanative framework is constructed for the systemic description and explanation of the phenomenon. To test the validity of the frame, verb-complement constructions are analyzed as a case study. It turns out that the constructions with disorienting complements have varying syntactic and semantic features from those with co-orienting complements. It also shows that the disorienting verb-complement constructions are motivated both linguistically and non-linguistically.

\section{REFERENCES}

[1] Bloomfield, L. (1933). Language. London: Allen \& Unwin.

[2] Chen W. D. (1932/2005). An Introduction to Rhetoric. Shanghai: Fudan University Press.

[3] Cong, Y. X. (2013). Mismatched modification in Chinese and English: A Grammatical Metaphor Perspective. PhD dissertation, Shanghai International Studies University.

[4] Croft, W. (2001). Radical Construction Grammar: Syntactic Theory in Typological Perspective. Oxford: Oxford University Press.

[5] Dai, H. Y. (1982). Additional discussion of "Rere de he yi wan cha". Journal of Chinese Linguistics (10): 81-85.

[6] Du, S. H. (2020). Description and Explanation in Language Studies. Contemporary Language Studies (4): 27-36.

[7] Gu, Y. (1992). The syntax of resultative and causative compounds in Chinese. PhD. Dissertation, Cornel University.

[8] Guo, R. (2002). Argument structure of verb-result structures. In Xu, L. J. \& Shao, J. M. (eds.) New Development of Chinese Grammar Studies (1). Hangzhou: Zhejiang Education Press.

[9] Jackendoff, R. (1972). Semantic Interpretation in Generative Grammar. Massachusetts: MIT Press.

[10] Jiang, L. (2006). Semantic and Syntactic Analysis of Complex Verb-Result constructions. Journal of Central China University of Science and Technology (Social Science Edition) (04): 66-70.

[11] Keiichiro O. (1982). Adjective movement in variable verb sentences. In Study of Sub-terms. Tokyo: Meiji Shoin.

[12] Li, L. D. (1984). What "complements" really modify? Chinese Learning (2): 15-24.

[13] Li, Y. C., Liu, Z. G., Liu, Z. M. (2014). Subjectivization and the semantic disorientation of adjectives in modern Chinese. Foreign Language Teaching and Research (3): 351-363+479.

[14] Lu J. M. (2013). Modern Chinese Grammar Research Course. Beijing: Peking University Press.

[15] Lu, J. (2003). An analysis on the syntactic realization and function of Chinese depictive adverbial modifiers. Studies in Language and Linguistics (1): 99-106.

[16] Lu, J. M. (2001). On semantic orientation analysis. In Ji, X. L. (eds.) Selected Works of Lu Jianming (pp.: 380-400). Changchun: Northeast Normal University Press.

[17] Lu, J. M. (2016). A study of language information structure. Journal of Han and Tibetan Languages (9): 201-4.

[18] Lv, S. X. (1986). The flexibility of Chinese syntax. Studies of the Chinese Language (1): 1-9.

[19] Ma, Z. \& Lu, J. M. (1997). A study of adjectival complements. Chinese Language Learning (1): 3-7.

[20] Masayuki N. (1987). The intention and result of Chinese and Japanese adjectives. Japanese Studies (10):5-15.

[21] Pan, X. D. (1981). A Survey of attribute movement. Studies of The Chinese Language (4): 277-8.

[22] Sapir, Edward. (1921). Language: An Introduction to the Study of Speech. New York: Harcourt, Brace \& World.

[23] Shao, J. M. (2002). The study of attribute movement from the perspective of three levels of word sequence. In Selected Works of Well-known Middle-aged Linguists. Hefei: Anhui Education Press, 110-29.

[24] Shao, J. M. (2007). A Study of Chinese Grammar. Beijing: The Commercial Press. 
[25] Shen J. X. (1999). Grammaticalization and the Skewed Relationship between Form and Meaning. In New Development of Chinese Linguistics. Hong Kong: City University of Hong Kong Press.

[26] Shen Y. (2008). Semantic orientation theory and Chinese syntactic components. In Shen Y. \& Feng S. L. (eds.) Contemporary Linguistic Theories and Chinese Studies. Beijing: The Commercial Press, 321-40.

[27] Shen, J. X. (2004). The grammar and semantics of the "zhuilei" construction. Language Science (06): 3-15.

[28] Shi, Y. Z. (2003). The Establishment of Modern Chinese Grammar System: The Emergence and Influence of Verb-Complement Structures. Beijing: Beijing Language and Culture University Press.

[29] Talmy, L. (2000). Toward a Cognitive Semantics. Cambridge: The MIT Press.

[30] Ungerer F. \& H. J., Schmid. (2006). An Introduction to Cognitive Linguistics. London: Longman.

[31] Yuan, Y. L. (2001). The control- reductive analysis of verb-result structures. Studies of the Chinese Language (5): $399-410+479$.

[32] Zhang, G. X. (2005). The principles of the semantic orientation of properties and the pragmatic motivation of syntactic movement. Studies of the Chinese Language (1): 16-28.

[33] Zhang, G. X. (2006). A Functional and Cognitive Research on Modern Chinese Adjectives. Beijing: The Commercial Press.

[34] Zhao Y. R. (1979). Spoken Chinese Grammar (translated by Lv S. X.). Beijing: The Commercial Press.

[35] Zheng G. Y. (1995a). Verb and subject orientated adverbials. Chinese Language Learning (3): 19-25.

[36] Zheng G. Y. (1995b). The verb-object orientated "making" adjectival adverbials. Chinese Language Learning (6): 23-7.

[37] Zheng G. Y. (1998). Verb-object orientated "visual perception" adjectival adverbials. Chinese Language Learning (1): 3-5.

[38] Zheng G. Y. (1999). Verb-object orientated "taste perception" adjectival adverbials. Oriental Forum (Journal of Qingdao University) (4): 74-6.

[39] Zheng G. Y. (2000). The orientation of adverbial adjectives in Mandarin. Wuhan: Central China Normal University Press.

[40] Zhu, D. X. (1982). Lectures on Grammar. Beijing: The Commercial Press.

Ting Yang received her Master's degree in English Language and Literature from Southwest University, China in 2012. She is currently a lecturer in the School of Foreign Languages, Chongqing College of Humanities, Science and Technology. Her research interests include Cognitive Linguistics, Second Language Acquisition and higher education. 